07,01

\title{
Влияние ультрамелкозернистой структуры материала на прочностные характеристики сплава алюминия при ударных нагрузках
}

\author{
(C) А.Д. Евстифреев, И.В. Смирнов, Ю.В. Петров \\ Санкт-Петербургский государственный университет, \\ Санкт-Петербург, Россия \\ E-mail: ad.evstifeev@gmail.com
}

Поступила в Редакцию 26 декабря 2018 г.

В окончательной редакции 23 января 2019 г.

Принята к публикации 23 января 2019 г.

Рост скоростей автомобильного и аэрокосмического транспорта одновременно с необходимостью снижения их массы приводит к росту требований к эксплуатационной надежности используемых материалов и конструкций. При этом работа материала в условиях динамических ударных нагрузках остается в ненормативных диапазонах. В работе представлена комплексная методика исследования и предсказания поведения материалов в условиях растягивающего и динамического воздействий. На примере алюминиевых сплавов систем $\mathrm{Al}-\mathrm{Mg}$ и $\mathrm{Al}-\mathrm{Cu}-\mathrm{Mg}$ показана возможность повышения прочностных свойств материала методами интенсивной пластической деформации (ИПД) в широком диапазоне изменения параметров внешнего воздействия и фиксация этих изменений в рамках предложенной методики.

Работа выполнена при поддержке гранта РНФ (№ 17-79-10145). Механические и структурные исследования проведены с использованием оборудования лаборатории СПбГУ „Механики перспективных массивных наноматериалов для инновационных инженерных приложений“, ресурсных центров Научного парка СПбГУ „Исследование экстремальных состояний материалов и конструкций“, „Нанотехнологии“ и „Рентгенодифракционные методы исследования“.

DOI: 10.21883/FTT.2019.06.47690.347

\section{1. Введение}

Благодаря уникальному сочетанию свойств алюминиевые сплавы широко применяются в автомобильной, авиационной и космической промышленности. Например, магналии, сплавы алюминия и магния, обладают хорошей прочностью и обрабатываемостью, а также высокой коррозионной стойкостью, благодаря чему хорошо зарекомендовали себя для изготовления обшивок на транспорте, а также для изготовления сосудов для транспортировки нефти и других химических веществ. Дюралюмины, сплавы алюминия, магния и меди, обладают повышенной прочностью, поэтому применяются в качестве конструкционных сплавов в автомобилях, самолетах и космических кораблях.

Широкое применение материалов на основе алюминия, в том числе на транспорте и в авиакосмической отрасли, требует всестороннего изучения прочностных характеристик материала в широком диапазоне изменения параметров внешнего нагружения. Особое внимание стоит уделять работе материалов в экстремальных условиях, поскольку с повышением скорости деформирования реакция материала существенно изменяется, повышается предельная прочность материала, связанная со структурно-временными особенностями процесса разрушения [1-6]. Это требует экспериментального и теоретического изучения динамических прочностных свойств.
Одним из наиболее часто применяемых методов исследования прочностных свойств материала в условиях ударных нагрузок является метод Кольского с использованием разрезных стержней Гопкинсона и его модификаций $[7,8]$. Стоит отметить, что данная методика основана на уникальном оборудовании и имеет определенные сложности в реализации. В настоящей работе предложена методика выполнения экспериментов на растяжение с использованием башенного копра, имеющего сертифицированные системы фиксации скорости ударника и усилия сопротивления образца растяжению.

На примере сплавов в состоянии поставки Амг6 системы Al-Mg (аналог 5556 ASTM) и Д16 системы $\mathrm{Al}-\mathrm{Cu}-\mathrm{Mg}$ (аналог $2024 \mathrm{ASTM}$ ) получены скоростные зависимости максимальных растягивающих напряжений для растягивающей и сдвиговой моды деформирования, а также показаны возможности повышения прочностных свойств материала методами интенсивной пластической деформации (ИПД) [9-11].

\section{2. Материал и методика экспериментальных исследований}

\section{1. Материал}

Изучение прочностных и эксплуатационных свойств материала является в значительной мере условным, 
Химический состав алюминиевых сплавов A15556 и A12024

\begin{tabular}{c|c|c|c|c}
\hline Элемент (mass.\%) & $\mathrm{Cu}$ & $\mathrm{Mg}$ & $\mathrm{Mn}$ & $\mathrm{Al}$ \\
\hline $\mathrm{A} 15556$ & $<0.1$ & $5.8-6.8$ & $0.5-0.8$ & Основа \\
$\mathrm{A} 12024$ & $3.8-4.9$ & $1.2-1.8$ & $0.3-0.9$ & Основа
\end{tabular}

поскольку однии те же материалы в различных условиях нагружения способны демонстрировать разный характер разрушения. Факторы, влияющие на разрушение, подразделяют на внутренние и внешние. К первым относятся химический состав и структура (размер зерна, степень разориентации, тип кристаллической решетки и т.д.). Из внешних факторов наиболее важными являются скорость нагружения и температура. Важными факторами являются также особенности напряженнодеформированного состояния и геометрические особенности исследуемых объектов.

Таким образом, переходя к изучению прочностных свойств материала в условиях широкого спектра нагрузок необходимо наиболее полно охарактеризовать их состояние с точки зрения стандартных подходов.

Рассмотрим широко используемые алюминиевые сплавы A15556 системы Al-Mg и A12024 системы $\mathrm{Al}-\mathrm{Cu}-\mathrm{Mg}$. Химический состав сплавов приведен в таблице. В сплаве A15556 магний является основным легирующим элементом. Будучи сплавом на основе твёрдого раствора он в закаленном состоянии имеет гомогенную структуру, что обеспечивает высокие пластические свойства. Последнее обстоятельство позволяет применять эти сплавы для тяжёлонагруженных деталей, работающих в условиях динамических нагрузок. В сплаве Al2024 медь является основным легирующим элементом. Сплавы этой серии широко используются в авиастроении, где требуется высокая прочность.

Микроструктура сплава A15556 состоит из зерен со средним размером $10 \mu \mathrm{m}$. Микроструктура сплава Al2024 состоит из зерен со средним размером $23 \mu \mathrm{m}$.

Первичный анализ механических свойств показал, что микротвердость по Виккерсу у сплава A12024 $(147.1 \pm 3.6 \mathrm{Hv})$ на $30 \%$ больше, чем у сплава $\mathrm{Al} 5556$ $(113.2 \pm 2.7 \mathrm{Hv})$. Предел кратковременной прочности на разрыв также отличается примерно на 30\%: $430 \pm 13$ и $319 \pm 11 \mathrm{MPa} \mathrm{соответственно.} \mathrm{С} \mathrm{точки} \mathrm{зрения} \mathrm{поведения}$ материалов в условиях квазистатических нагрузок, где требуется повышенная прочность, наиболее предпочтительным является сплав A12024. При этом у сплава A15556 есть преимущества, описанные выше. С целю повышения его прочностных свойств была выполнена обработка методами ИПД. Для формирования ультрамелкозернистой (УМЗ) структуры исходные заготовки были подвергнуты деформационной обработке. При равноканальном угловом прессовании (РКУП) [12-14] был использован маршрут $\mathrm{Bc}$, при котором после каждого из четырех проходов заготовка поворачивается на $90^{\circ}$ вокруг своей продольной оси. В процессе прессования при температуре $200^{\circ} \mathrm{C}$ материал продавливался в специальной оснастке через два канала диаметром $9.5 \mathrm{~mm}$, пересекающихся под углом $90^{\circ}$.

Ультрамелкозернистый материал продемонстрировал повышение микротвердости по Виккерсу $149.2 \pm 2.8 \mathrm{Hv}$. Предел кратковременной прочности также повысился до уровня прочностных характеристик сплава Al2024 $430 \pm 10 \mathrm{MPa}$. Микроструктура сплава A15556 после четырех проходов РКУП стала более однородная и состоит из зерен со средним размером $2 \mu \mathrm{m}$, что также подтверждается данными из работы [15].

\section{2. Методики квазистатических и динамических испытаний на растяжение}

Известно, что для многих материалов, при увеличении скорости деформации критическое напряжение растет [16-19]. В настоящей работе для выполнения экспериментов на растяжение были применены серийные экспериментальные установки. Установка Shimadzu AG-50kNX использовалась для растяжения в квазистатических режимах, а установка Instron CEAST 9350 для реализации ударных растягивающих нагрузок при скорости деформации порядка $10^{2}-10^{3} \mathrm{~s}^{-1}$.

Выполненные ранее работы [20,21] показали работоспособность методики испытаний на растяжение малых образцов с длиной рабочей части $5 \mathrm{~mm}$, шириной $2 \mathrm{~mm}$. В этом случае реализуется одноосное растягивающее напряженное состояние.

Другим важным аспектом в изучении прочностных свойств конструкционных материалов является исследования прочности на сдвиг. Как вид напряженного состояния, встречается редко, но может реализовываться в ответственных элементах и узлах, к примеру, может иметь место в заклепочных и сварных соединениях.

Для реализации сдвиговых напряжений при растяжении была подобрана геометрия (рис. $1, a)$, аналогичная представленной в [22,23], адаптированная к возможностям испытательных машин и геометрическим размерам образцов из материалов, подвергнутых равноканальному угловому прессованию. По полученным в ANSYS данным (рис. $1, b)$ видно, что в узкой области между круговыми отверстиями при растяжении реализуются локализованные поля сдвиговых деформаций. Это также подтверждается микроструктурными исследованиями разрушенных образцов. На рис. 2 представлена фрактография разрушенного образца при увеличении $5 \times$ и $20 \times$, соответственно. При масштабе $200 \mu \mathrm{m}$ видно, что поля сдвиговых деформаций не выходят за границу локального объема, ограниченного круговыми вырезами. В то время как при увеличении $20 \times$ в выделенном объеме наблюдаются сплошные полосы сдвига.

\section{3. Результаты и дискуссия}

Предел кратковременной прочности для исследуемых материалов для двух типов реализуемых деформаций 


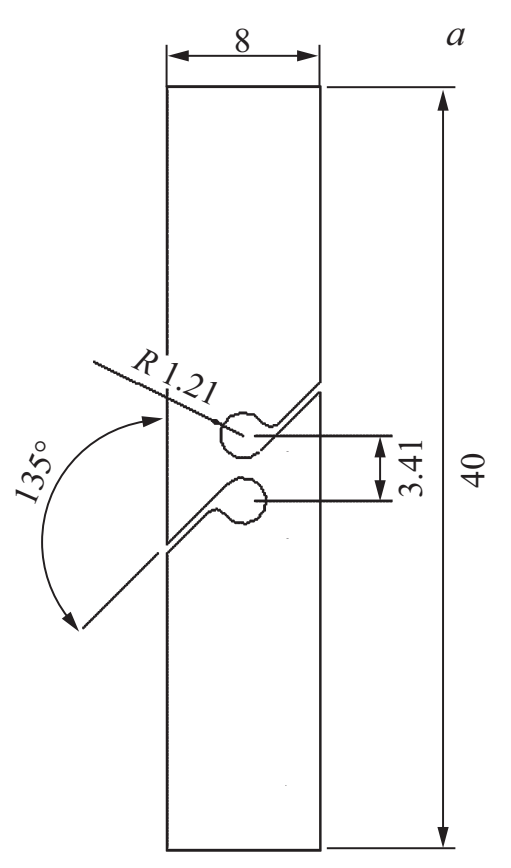

A: static structural

Shear elastic strain

Type: shear elastic strain ( $X Y$ plane) - top/bottom

Unit: $\mathrm{m} / \mathrm{m}$

Global coordinate system

Time: 1

28.10.2018 $20: 28$

$0.020631 \mathrm{max}$

0.015127

$-0.0096217$

$-0.0041168$

$-0.0013881$

$-0.006893$

$-0.012398$

$-0.017903$

$-0.023408$

$-0.028913 \mathrm{~min}$

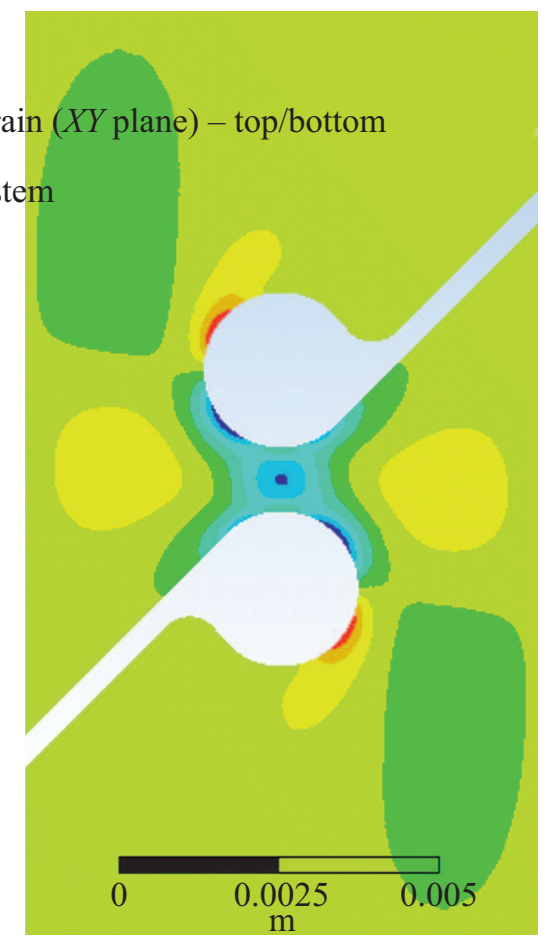

$b$

Pис. 1. Геометрические размеры образца на сдвиг (а). Рассчитанные в ANSYS поля сдвиговых деформаций при растягивающей нагрузке $500 \mathrm{~N}(b)$.
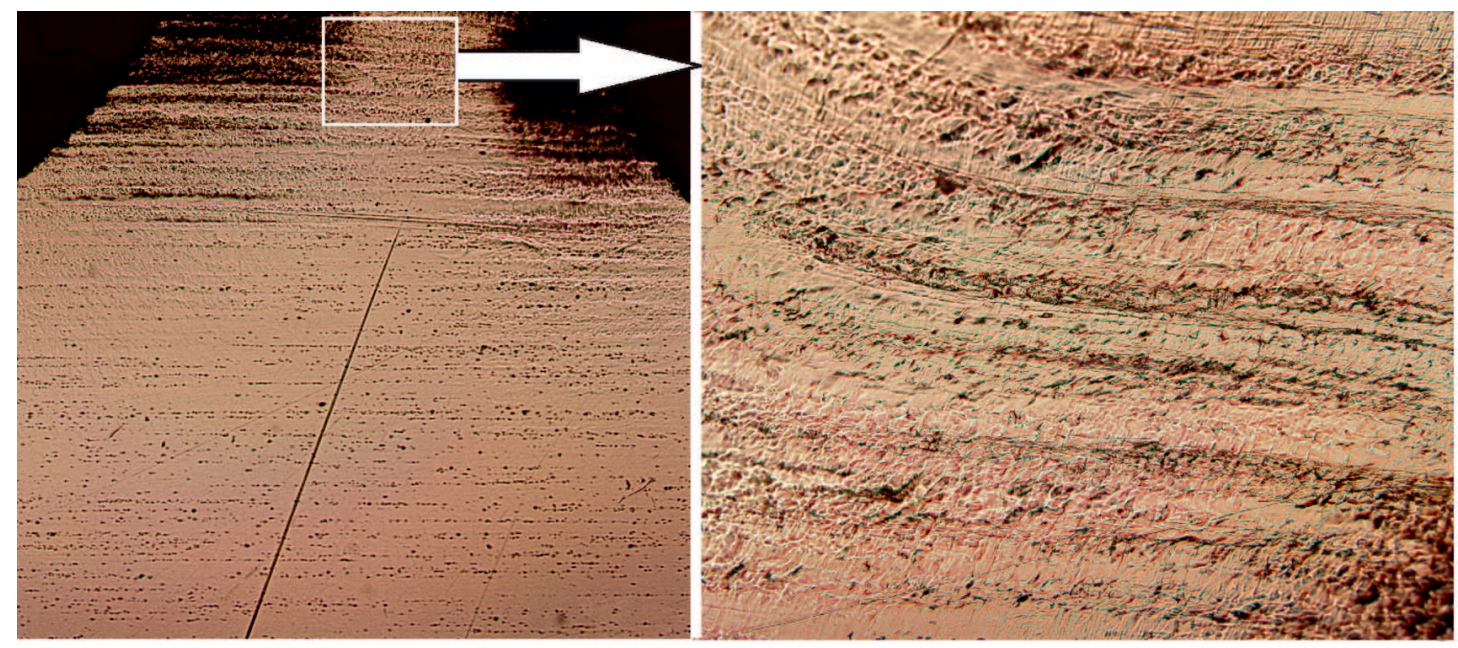

$-200 \mu \mathrm{m}$

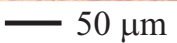

Рис. 2. Поля сдвиговых деформаций в образцеиз А15556 после испытания.

(растяжение/сдвиг) в зависимости от скорости роста напряжений представлены на рис. 3. Скоростную зависимость прочности определяли относительно скорости роста напряжений. Данный подход позволил минимизировать влияние погрешностей, связанных с жесткостью испытательной машины и свойств материала. Полученные результаты демонстрируют, что в условиях квазистатических нагрузок сплав Al5556 имеет меньшую прочность при растяжении и сдвиге, чем сплав Al2024.
Квазистатические режимы деформирования соответствуют скоростям роста напряжений $50-100 \mathrm{GPa} / \mathrm{s}$. C ростом скорости приложения нагрузки при реализации схемы на сдвиг соотношение сохраняется, в то время как в случае динамического растяжения происходит сближение экспериментальных точек.

Отмеченные особенности поведения материала при высоких скоростях нагружения можно объяснить с применением структурно временного подхода. В качестве 


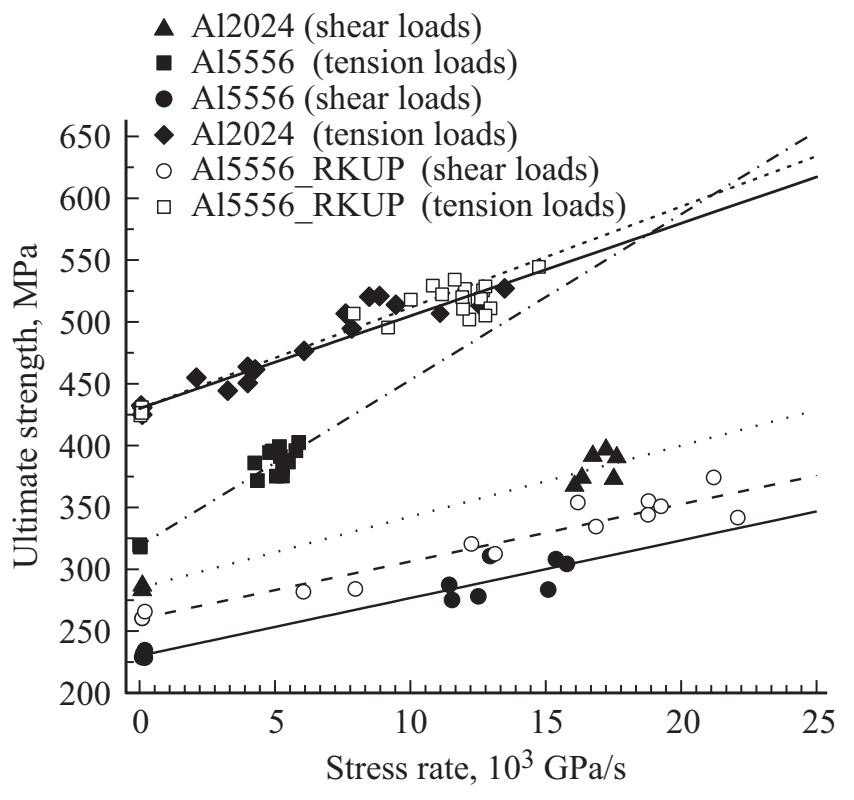

Рис. 3. Зависимость прочностина растяжение и сдвиг от скорости роста напряжений для алюминиевых сплавов A12024, Al5556 и A15556 после РКУП обработки. Динамические кривые для каждого вида реализуемой нагрузки и материла построены по критерию (1) с параметрами материала: для сплава А12024 при растяжении $\tau_{c}=17.5 \mu \mathrm{s}, \sigma_{c}=430 \mathrm{MPa}$ при сдвиге $\tau_{c}=11.5 \mu \mathrm{s}, \sigma_{c}=285 \mathrm{MPa}$; для сплава $\mathrm{A} 15556$ при растяжении $\tau_{c}=26.8 \mu \mathrm{s}, \sigma_{c}=319 \mathrm{MPa} \mathrm{при} \mathrm{сдвиге} \tau_{c}=9.3 \mu \mathrm{s}$, $\sigma_{c}=230 \mathrm{MPa}$; для сплава Al5556 после РКУП обработки при растяжении $\tau_{c}=15 \mu \mathrm{s}, \sigma_{c}=430 \mathrm{MPa} \mathrm{при} \mathrm{сдвиге} \tau_{c}=9.3 \mu \mathrm{s}$, $\sigma_{c}=260 \mathrm{MPa}$.

критерия разрушения материала при растяжении используем критерий инкубационного времени $[24,25]$

$$
\frac{1}{\tau_{c}} \int_{t-\tau_{c}}^{t} \frac{\sigma(s)}{\sigma_{c}} d s \leq 1,
$$

где $t-$ время, $\sigma$ - зависимость разрывающего (растяжение/сдвиг) напряжения от времени, $\sigma_{c}-$ статический предел прочности при растяжении или сдвиге, $\tau_{c}$ - инкубационное время разрушения, являющейся мерой прочности в динамическом диапазоне параметров внешнего воздействия. Константы $\sigma_{c}$ и $\tau_{c}$ являются параметрами материала. В качестве модельной нагрузкипринимается - линейно возрастающая во времени, что наиболее приближенно к условиям эксперимента.

Анализируя полученные данные можно отметить, что алюминиевые сплавы A12024 и A15556 имеют примерно одинаковую динамическую прочность на сдвиг в терминах инкубационного времени. Это выражается в наклоне расчетной кривой в координатах предельное напряжение-скорость роста напряжений. И тем самым, с ростом скорости приложения нагрузки тенденция относительно соотношения прочностных характеристик двух материалов, полученная в квазистатической области нагружения, сохраняется. В случае же реализации растягивающей нагрузки динамические кривые прочности пересекаются и происходит инверсия прочности. При квазистатических нагрузках сплав A12024 примерно на $30 \%$ прочнее сплава Al5556. С ростом скорости нагружения сплав A12024 теряет свое преимущество и может разрушиться при меньших нагрузках, нежели сплав A15556. Это связано с динамической прочностью в терминах инкубационного времени. У сплава A15556 $\tau_{c}=26.8 \mu \mathrm{s}$, в то время как для сплава A12024 эта величина равняется $16.3 \mu \mathrm{s}$.

Обработка методами РКУП алюминиевого сплава A15556 привела к повышению статической прочности на сдвиг и на растяжение. При сдвиговых нагрузках динамическая прочность материала не изменилась, а статическая повысилась примерно на 10\%. При растяжении с реализацией сдвиговой моды разрушения ультрамелкозернистый материал продемонстрировал повышение статической прочности до уровня сплава A12024 с сохранением динамической прочности на высоком уровне.

\section{4. Заключение}

Разработана методика комплексных испытаний материла в широком диапазоне изменения параметров внешнего воздействия - от квазистатического нагружения до высокоскоростного ударного воздействия. Полученные результаты в рамках структурно временного подхода позволяют проводить сравнительный анализ материалов в условиях динамических воздействий.

Апробация методики была проведена на примере широко используемых алюминиевых сплавов системы $\mathrm{Al}-\mathrm{Cu}-\mathrm{Mg}$ и $\mathrm{Al}-\mathrm{Mg}$. Результаты экспериментальных работ показали повышенные прочностные свойства сплава A12024 в условиях квазистатических нагрузок. С ростом скорости нагружения в случае растягивающих нагрузок данный сплав по своим прочностным характеристикам приблизился к сплаву A15556. При этом в случае реализации чистого сдвига запас по прочности сохранился. Полученные результаты были проанализированы с точки зрения структурно временного подхода. Получено, что в терминах инкубационного времени динамическая прочность при растяжении у сплава A15556 на $65 \%$ больше, чем у сплава Al2024, что объясняет пересечение прочностных кривых.

Модификация структуры сплава A15556 путем равноканального углового прессования позволило повысить статическую прочность материала при растяжении до уровня сплава A12024 с сохранением динамической прочности на высоком уровне, что было зафиксировано в рамках численных расчетов.

Выполненные исследования показали, что применение материала в экстремальных условиях нагружения требует всестороннего экспериментально-теоретического изучения его прочностных и эксплуатационных свойств в предполагаемых диапазонах изменения параметров нагружения. 


\section{Список литературы}

[1] G.V. Stepanov, V.V. Astanin, V.I. Romanchenko, A.P. Vashchenko, V.M. Tokarev, B.D. Chukhin, Y.P. Guk. Strength Mater. 15, 220 (1983).

[2] А.М. Брагов, А.К. Ломунов. ПМТФ 5, 168 (1988).

[3] A.A. Gruzdkov, E.V. Sitnikova, N.F. Morozov, Y.V. Petrov. Mathem. Mech. Solids 14, 72 (2009).

[4] A.A. Gruzdkov, S.I. Krivosheev, Y.V. Petrov. Phys. Solid State 45, 886 (2003).

[5] G.I. Kanel, S.V. Razorenov, A.A. Bogatch, A.V. Utkin, V.E. Fortov, D.E. Grady. J. Appl. Phys. 20, 467 (1997).

[6] Г.В. Гаркушин, Г.И. Канель, С.В. Разоренов. ФТТ 52, 2216 (2010).

[7] H. Kolsky. Proc. Phys. Soc. B 62, 676 (1949).

[8] A.M. Bragov, A.K. Lomunov. Int. J. Impact Eng. 16, 321 (1995).

[9] R. Valiev. Nature Mater. 3, 511 (2004). Q. Wei. Acta Mater. 54, 4079 (2006).

[10] L.W. Meyer, M. Hockauf, L. Kruger, I. Schneider. Int. J. Mater. Res. 98, 191, (2007).

[11] M.A. Meyers, A. Mishra, D.J. Benson. Progr. Mater. Sci. 51, 427 (2006).

[12] R.Z. Valiev, R.K. Islamgaliev, I.V. Alexandrov. Progr. Mater. Sci. 45, 103 (2000).

[13] I.P. Semenova, M.K. Smyslova, K.S. Selivanov, R.R. Valiev, Y.M. Modina. In IOP Conf. Ser.: Mater. Sci. Eng. 194, 012035 (2017).

[14] I.P. Semenova, A.V. Polyakov, V.V. Polyakova, Y. Huang, R.Z. Valiev, T.G. Langdon. Adv. Eng. Mater. 18, 2057 (2016).

[15] А.А. Козулин, В.А. Красновейкин, В.В. Скрипняк, Б.В. Хандаев, Ю.В. Ли. Современ. проблемы науки и образования 6, 888 (2013).

[16] W. Goldsmith, J.L. Sackman, C. Ewerts. Rock. Mech. Min. Sci. Geomech. 13, 303 (1976).

[17] S. Howe, W. Goldsmith, J. Sackman. Exp. Mech. 14, 337 (1974).

[18] Y.V. Petrov, I.V. Smirnov, A.A. Utkin. Mech. Solids 45, 476 (2010).

[19] Н.А. Златин, С.М. Мочалов, Г.С. Пугачев, А.М. Брагов. ФTT 16, 1752 (1974).

[20] A.D. Evstifeev, A.A. Chevrychkina, Y.V. Petrov. Mater. Phys. Mech. 32, 258 (2017).

[21] A.D. Evstifeev. J. Phys.: Conf. Ser. 991, 012019 (2018).

[22] M. Brünig, O. Chyra, D. Albrecht, L. Driemeier, M. Alves. Int. J. Plasticity 24, 1731 (2008).

[23] Q. Yin, B. Zillmann, S. Suttner, G. Gerstein, M. Biasutti, A.E. Tekkaya, A. Brosius. Int. J. Solids Struct. 51, 1066 (2014).

[24] Y.V. Petrov, A.A. Utkin. Sov. Mater. Sci. 25, 153 (1989).

[25] Y.V. Petrov, N.F. Morozov. ASME J. Appl. Mech. 61, 710 (1994).

Редактор К.В. Емцев 